\title{
Multidimensional Assessment of the Effectiveness of Group Voice Therapy
}

\author{
*Giovanna Cantarella, *+Sara Torretta, *Silvia Ferruta, *Annaclara Ciabatta, ¥Claudia Manfredi, \\ *,+Lorenzo Pignataro, and §Philippe Dejonckere, $* \dagger$ Milan and $\ddagger$ Firenze, Italy, and §Brussels, Belgium
}

\begin{abstract}
Summary: Objective. Group voice therapy has been successfully used in patients with dysphonia, but there is little objectively documented evidence of its effects on voice quality and the self-perception of voice fatigue. The aim of this study was to investigate the effectiveness of group therapy in patients with functional dysphonia and minor anatomic vocal fold pathologies linked by appearance and history to voice use in an objective and multidimensional manner.

Study design. This is a prospective longitudinal study.

Methods. Before and after treatment, 34 adult women underwent perceptual voice assessments using the grade of dysphonia, roughness, breathiness, asthenia, and strain (GRBAS) scale, Evaluation Vocale Assistée (EVA) system aerodynamic and acoustic assessments, and maximum phonation time measurements, and made subjective evaluations using the Voice Handicap Index. The pretreatment baseline values of the participants were obtained by means of two examinations separated by an interval of 1 week. The parameters belonging to each main dimension were clustered by means of $Z$-transformation, and the corresponding $Z$-scores were analyzed.

Results. Group therapy was associated with a statistically significant improvement in the perceptual $(P=0.008)$, acoustic $(P=0.040)$, aerodynamic $(P=0.009$ and $<0.001)$, and self-evaluation parameters $(P=0.011)$.

Conclusions. Our findings provide evidence that group voice therapy can be associated with improvements in perceptual, acoustic, aerodynamic, and self-evaluated parameters in some patients with dysphonia. Controlled, randomized studies are needed in follow-up. This method of treatment may be a means of reducing the costs and waiting lists associated with rehabilitative treatment, and enhancing patients' motivation and compliance.
\end{abstract}

Key Words: Dysphonia-Voice fatigue-Group voice therapy-Voice-Multidimensional assessment.

\section{INTRODUCTION}

A number of studies have confirmed the effectiveness of voice therapy in the treatment of nonorganic dysphonia and benign vocal fold lesions, but the significant objective improvements that can be obtained need to be documented using a multidimensional approach. ${ }^{1}$ Individual voice therapy sessions are the standard of care for patients with dysphonia, but can be a considerable economic burden on national health and social welfare services, as well as on subjects paying for the sessions themselves.

However, although there is still little objective evidence concerning its effects on voice quality and the self-perception of voice fatigue, group voice therapy has been successfully used in patients with voice and speech disorders, ${ }^{2}$ and may also have the advantage of optimizing voice therapists' working time and, by allowing a larger number of patients to be treated, reducing the length of waiting lists and increasing the efficient use of national health service resources. Providing group voice therapy sessions for patients who are homogeneous in terms of age, and the type and severity of their voice disorder, might stimulate mutual help and emulation in acquiring correct vocal behavior and improving voice quality. Reciprocal psychological support might also help the acquisition of motor skills and further

\footnotetext{
Accepted for publication February 10, 2017.

From the *Otolaryngology Department, Fondazione IRCCS Ca' Granda Ospedale Maggiore Policlinico, Milan, Italy; †Department of Clinical Sciences and Community Health, Università degli Studi di Milano, Milan, Italy; $\ddagger$ Department of Information Engineering, Università degli Studi di Firenze, Firenze, Italy; and the $\S$ Dept. of Neurosciences, University of Leuven and Federal Agency for Occupational Risks, Brussels, Belgium.

Address correspondence and reprint requests to Giovanna Cantarella, Otolaryngology Department, Fondazione IRCCS Ca' Granda Ospedale Maggiore Policlinico, Milan, Italy.

E-mail: giovanna.cantarella@policlinico.mi.it

Journal of Voice, Vol. 31, No. 6, pp. 714-721

0892-1997

(C) 2017 The Voice Foundation. Published by Elsevier Inc. All rights reserved.

https://doi.org/10.1016/j.jvoice.2017.02.004
}

motivate the patients. The effectiveness of group therapy has been widely demonstrated in the field of psychiatry for the treatment of mental disorders ${ }^{3,4}$; although psychiatric disorders are a very different condition from dysphonia, the reported positive outcomes show that patients are facilitated in understanding, following the therapist, and developing skills. Another field of application of group therapy with positive reports has been physical therapy for neurologic disorders such as Parkinson's disease and for the treatment of disabilities consequent to stroke. ${ }^{5}$ The group sessions of rehabilitation provided more behavioral changes than the individual treatment sessions in patients affected by Parkinson's disease. ${ }^{5}$ After stroke, the group approach allowed a more efficient time use and positive effects on the outcomes due to enhanced motivation, social interaction and reinforcement, and competition among the patients. ${ }^{6}$

The aim of the present study was to assess the effectiveness of group therapy in changing vocal behavior and improving voice quality in patients diagnosed with functional dysphonia with or without minor anatomic vocal fold pathologies by appearance and history presumed due to voice use using a multidimensional protocol including perceptual, acoustic, aerodynamic, and self-evaluation parameters.

\section{Study design and setting}

This prospective longitudinal study, which was approved by our local institutional review board and conducted in accordance with the standards of good clinical practice and the principles of the Helsinki Declaration, was carried out by the members of the voice therapy service of Fondazione IRCCS Ca' Granda Ospedale Maggiore Policlinico in Milan between January and December 2015. All patients gave their written informed consent. 


\section{Study subjects}

The study involved 34 adult women (mean age $40.9 \pm 14.0$ years, range 22-70) who were candidates for rehabilitation treatment for a voice disorder and vocal fatigue due to functional or minor organic changes in the vocal folds (vocal fold nodules or mild edema). The inclusion criteria were female gender, $\geq 18$ years of age, dysphonia and vocal fatigue due to functional causes, with possible tissue reaction to phonotrauma confirmed by videolaryngostroboscopy, and an indication for voice therapy. Only women were included to have a homogeneous group of patients, and also because the vast majority of patients seeking voice therapy for functional or minor organic causes in our service are women.

Thirteen of the patients (38\%) were school teachers, and all had a history of vocal overuse: 12 were affected by vocal fold nodules, 13 showed minor anatomic changes (vocal fold edema), and 9 had functional dysphonia only (muscle tension dysphonia or vocal folds bowing). Each patient was his or her own control, and all underwent a second multidimensional evaluation 1 week after the first to obtain pretreatment baseline values.

The exclusion criteria were laryngeal motility disorders, pathologies with an indication for phonosurgery, refusal to participate in group therapy, unfinished rehabilitation program, and previous phonosurgery or laryngeal radiotherapy.

\section{Outcomes}

At the enrollment visit (T0), a complete clinical history was taken and a record was made of each patient's age, profession, and extraprofessional vocal habits. Rigid videolaryngostroboscopy was used to detect the physical cause of dysphonia or vocal fatigue.

Upon enrollment (T0) and 1 week later (T1), to confirm the stability of the clinical features of the voice disorder, all patients underwent multidimensional evaluations.

\section{Multidimensional evaluation protocol}

A perceptual voice assessment was conducted using the GRBAS scale, ${ }^{7}$ which evaluates the overall grade of dysphonia, roughness, breathiness, asthenia, and strain in a voice. The voice samples were computer-recorded using a dynamic microphone (AKG, model B 29L, Acoustics, Vienna, Austria) at a constant distance of $5 \mathrm{~cm}$ from the patient's mouth (at an angle of about $60^{\circ}$ ) during the production of a sustained /a/, the repetition of single words and sentences (the Italian word "aiuole" and the sentence "Il bambino ama le aiuole della mamma"), and conversation. The patients were instructed to talk at a comfortable pitch and loudness. All of the voice samples were subsequently blindly evaluated by three independent listeners experienced in voice diseases (a voice therapist, an otolaryngologist, and a phoniatrician) and were scored as follows: $0=$ normal, 1 = slight disturbance, $2=$ moderate disturbance, and $3=$ severe disturbance. The perceptual scores were defined as the mean scores for $\mathrm{G}, \mathrm{R}$, and $\mathrm{B}$, as rated independently by the three listeners, blinded as regards the moment of the recording. In a last step, the mean $\mathrm{G}, \mathrm{R}$, and B scores were averaged to obtain a final global perceptual score.

Aerodynamic and acoustic assessments of voice recordings were made using the Evaluation Vocale Assistée (EVA) system (SQ-Lab, Aix-en-Provence, France). ${ }^{8}$ A rubber mask con- nected to a mouthpiece was placed over the patient's mouth and made to adhere closely to the skin to avoid any air leakage. The mouthpiece contained a calibrated directional microphone at a distance of $2 \mathrm{~cm}$ from the patient's oral opening and a grid pneumotachograph; the microphone and aerodynamic sensor were coaxial so that voice sound and phonatory airflow could be simultaneously recorded. The two assessments were as follows:

1. A simultaneous acoustic and aerodynamic evaluation derived from the patient's production of a sustained /a/ at a comfortable pitch and intensity for at least 4-5 seconds. A 1-second segment of the most stable part of the signal was selected for analysis. ${ }^{9}$ The acoustic indices considered were percent jitter (computed as the mean absolute difference between the periods of adjacent cycles divided by the mean period; the proportion is then multiplied by 100 to get a percentage), percent shimmer (obtained similarly using peak-to-peak amplitudes), and the harmonicsto-noise ratio (HNR, in which $\mathrm{H}$ is the energy of the averaged wave form, and $\mathrm{N}$ is the mean energy of the differences between the individual periods and the averaged wave form) expressed in decibel. ${ }^{10}$

2. An airway-interruption method for indirectly estimating subglottic pressure and phonation threshold pressure (PTP). ${ }^{11}$ A pressure sensor placed in the oral cavity measured intraoral pressure during the emission of a sequence of "pa" from unvoiced to voiced. The patient was instructed to pronounce an uninterrupted sequence of 6-7/pa/ with a very short interval, first whispering and then gradually gaining sound in the voice. Then the subject pronounced a sequence of $/ \mathrm{pa} /$ at a comfortable pitch and loudness. Oral airflow, oral pressure, and acoustic signal were recorded simultaneously to obtain an estimated PTP and an estimated subglottic pressure during spontaneous phonation. ${ }^{12}$ The investigator ensured that airflow had gone to zero during /p/ occlusion before proceeding with the analysis. Then pressure peaks at the $/ \mathrm{p} /$ occlusion were carefully measured from the obtained tracings to estimate the PTP and the subglottic pressure during spontaneous phonation.

Three consecutive measurements of the maximum phonation time (MPT) were obtained from the sustained emission of the vowel /a/ at a comfortable pitch and volume; the longest of the three times was used in the data analysis. ${ }^{13}$ The investigator prompted the subject to achieve the best possible result after deep inspiration.

A subjective evaluation of patient-perceived vocal status was obtained using the Voice Handicap Index (VHI). ${ }^{14}$ The 30 items of the index were divided into three subscales that assess the functional, physical, and emotional aspects of the disability caused by the voice impairment. The subscale scores ranged from 0 to 40 , and the total score ranged from 0 to 120 ; a higher score indicates a greater degree of self-perceived disability.

The investigated parameters are the basic and classical issues in the field of voice function assessment and, although the different dimensions have shown poor redundancy, are not completely independent. ${ }^{15}$ 


\section{Group voice rehabilitation protocol}

Approximately 1 week after the baseline evaluations, the patients underwent seven 1-hour sessions of group voice rehabilitation therapy: the first six sessions were held weekly, and the seventh took place 1 month after the sixth. The groups consisted of four or five patients selected on the basis of their similar age and cause of dysphonia. The same senior speech therapist (A.C.), experienced in voice disorders rehabilitation, performed all the rehabilitative sessions. After each session of voice therapy, the subjects were instructed to repeat all of the training exercises performed with the speech therapist during the session two to three times a day for 10-15 minutes depending on the severity of the voice impairment, and to make notes about the exercises in a daily diary. The main aims of the treatment were to improve the steadiness of the voice and to reduce breathiness while improving vocal fold closure by strengthening the adductor muscles and reducing vocal effort. ${ }^{16,17}$

The rehabilitation protocol follows a general scheme utilized in our service, with exercises traditionally utilized in our region. The main steps of the treatment included teaching of vocal hygiene principles, relaxation exercises, abdominal breathing training, resonance voice exercises such as humming to enhance the feeling of oral vibratory sensations, ${ }^{18}$ digital manipulation of the thyroid cartilage, neck muscle massage, articulation exercises, and semi-occluded vocal tract exercises such as lip and tongue drills. Special attention was paid to achieving and maintaining optimal respiratory and phonatory coordination during spontaneous speech, and care was taken to correct hyperfunctional phonatory behaviors.

One week after the end of the rehabilitation period (T2), all patients repeated the multidimensional evaluation described above.

\section{Statistical analysis}

The results are given as absolute numbers and percentages, or arithmetical mean values \pm standard error. The intraclass correlation coefficient (ICC) was computed to assess the inter-rater reliability as regards perceptual evaluation of $\mathrm{G}, \mathrm{R}$, and $\mathrm{B}$. The Wilcoxon nonparametric test for paired samples was used for comparing the patients' perceptual, acoustic, aerodynamic, and self-evaluation data. First, the two baseline measures were compared. In case of no significant difference, the two measures were averaged and a second test was performed to compare the average pretherapy value with the post-therapy measure.

The European Laryngological Society protocol ${ }^{19}$ considers five dimensions in voice assessment: perception, acoustics, aerodynamics, imaging (biomechanics), and patient self-evaluation. For this article, imaging is not relevant. Each of the four remaining dimensions comprises several possible parameters and measurements, and these are to some extent related to each other. For the present study, in line with the European Laryngological Society proposal, we clustered the measures (scores) within each dimension. However, as the units of the acoustic (jitter-shimmerHNR) and aerodynamic (MPT-PTP-subglottic pressure during spontaneous phonation) parameters were different, the raw values were first transformed or "standardized" into Z-scores, which has the effect of transforming the original distribution to one in which the mean becomes 0 and the standard deviation becomes
1 , and quantifying the original score as the number of standard deviations that the score is from the global mean. So we obtained a single final value for each dimension, and these values are suited for a comparison between the "baseline" (mean of the two measures before therapy) and the post-therapy status.

The Cohen $d$ was calculated as the pooled standard deviation between the mean pre- and post-treatment values to evaluate the impact of effect size (defined as small if $d \leq 0.3$, medium if $d$ is between 0.4 and 0.7 , and large if $d \geq 0.8$ ) on the treatment effect. ${ }^{20}$

Finally, a correlation matrix was used to present all of the computed correlation coefficients to evaluate the degree and direction of the associations between the variables, with correlation coefficients of +1 indicating a perfect direct relationship and those of -1 indicating a perfect inverse relationship.

The data were analyzed using SPSS 17.0 software (SPSS Inc, Chicago, IL), and a $P$ value of $<0.05$ was considered statistically significant.

\section{RESULTS}

All the patients successfully completed the group voice therapy protocol, the outcomes of which are shown in Figures $1-7$. The home diary that the patients kept apparently showed that they adhered to the request of repeating the exercises at home two to three times a day.

The demographic and main clinical characteristics of each voice group are reported in Table 1.

\section{Perceptual evaluation}

Figure 1 shows the average G, R, and B scores at each timepoint. The parameters $\mathrm{A}$ and $\mathrm{S}$ were not considered for statistics. The ICC was computed to assess the inter-rater reliability as regards perceptual evaluation of $\mathrm{G}, \mathrm{R}$, and $\mathrm{B}$. The ICC is a general measure of agreement or consensus. The coefficient represents agreement between several raters on the same set of data and is adjusted for the effects of the scale of measurements. The overall ICC for the three listeners was 0.89. Cicchetti ${ }^{21}$ considered an ICC of 0.75 or more as indicating "excellent." The

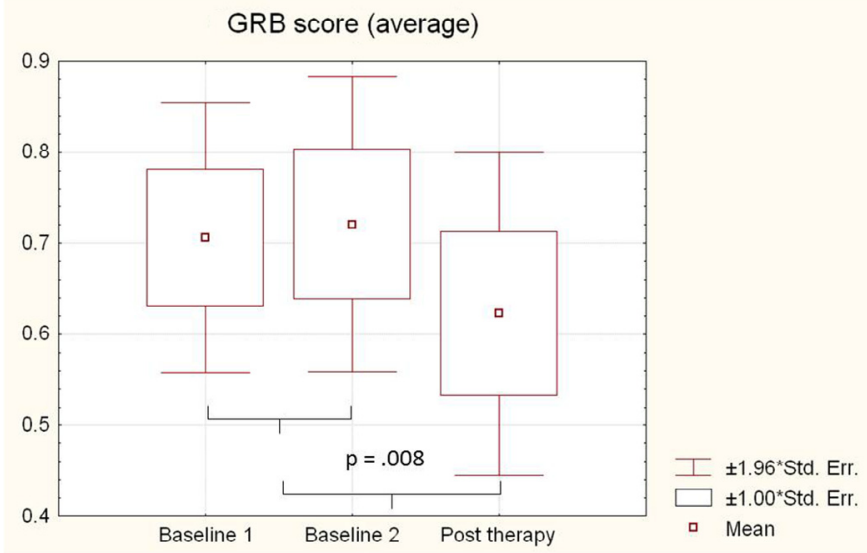

FIGURE 1. Differences between pre- and post-therapy GRB scores. GRB, grade of dysphonia, roughness, breathiness; SE, standard error. 


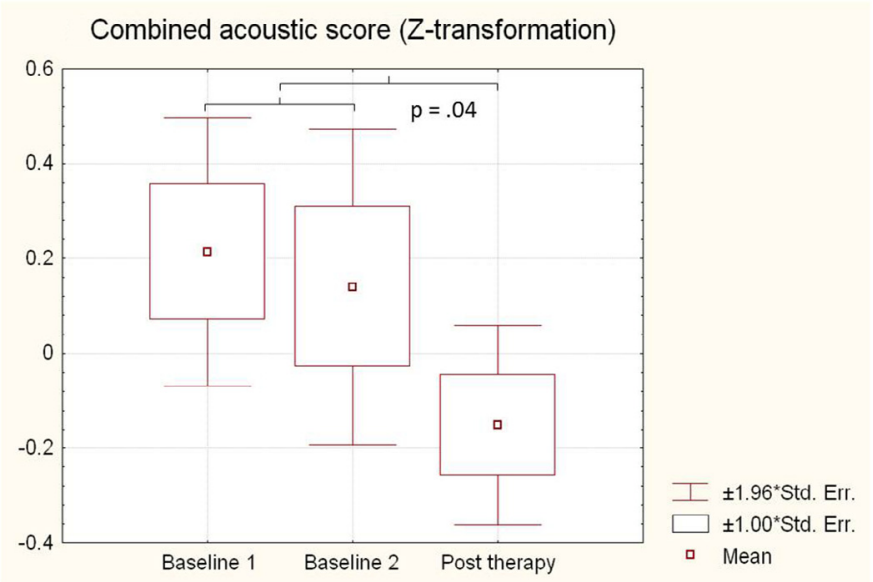

FIGURE 2. Differences between pre- and post-therapy Z-transformed combined acoustic scores. SE, standard error.

Wilcoxon test for paired values showed that the results of the two baseline assessments were not significantly different, but there was a statistically significant difference between the average baseline value and the post-treatment value $(P=0.008)$.

\section{Acoustic parameters}

Percent jitter and shimmer are proposed to decrease when the voice is improving, whereas the HNR should increase in case of improvement. Figure 2 shows the combined acoustic score obtained after Z-transformation, HNR sign inversion, and averaging. Once again, the Wilcoxon test for paired values showed that the two baseline measurements were not significantly different, but there was a statistically significant improvement between the average baseline value and the post-treatment value $(P=0.040)$.

\section{Aerodynamic parameters}

Estimated subglottic pressure during phonation and PTP have the same unit of measurement (hPa) but a different distribution, whereas MPT is expressed in seconds. Lower subglottic pressure and lower PTP during phonation, but longer MPT, imply better voice function. ${ }^{22-24}$ Figure $3 \mathrm{~A}$ shows the combined aerodynamic score obtained after $Z$-transformation, MPT sign inversion, and averaging. The Wilcoxon test for paired values showed a nonsignificant difference between the two baseline values but a highly significant difference between the average baseline value and the post-treatment value $(P<0.0001)$. Figures $3 \mathrm{~B}-\mathrm{D}$ and 4 show that the greatest changes were in MPT
Combined Aerodynamic score (Z-transformation)
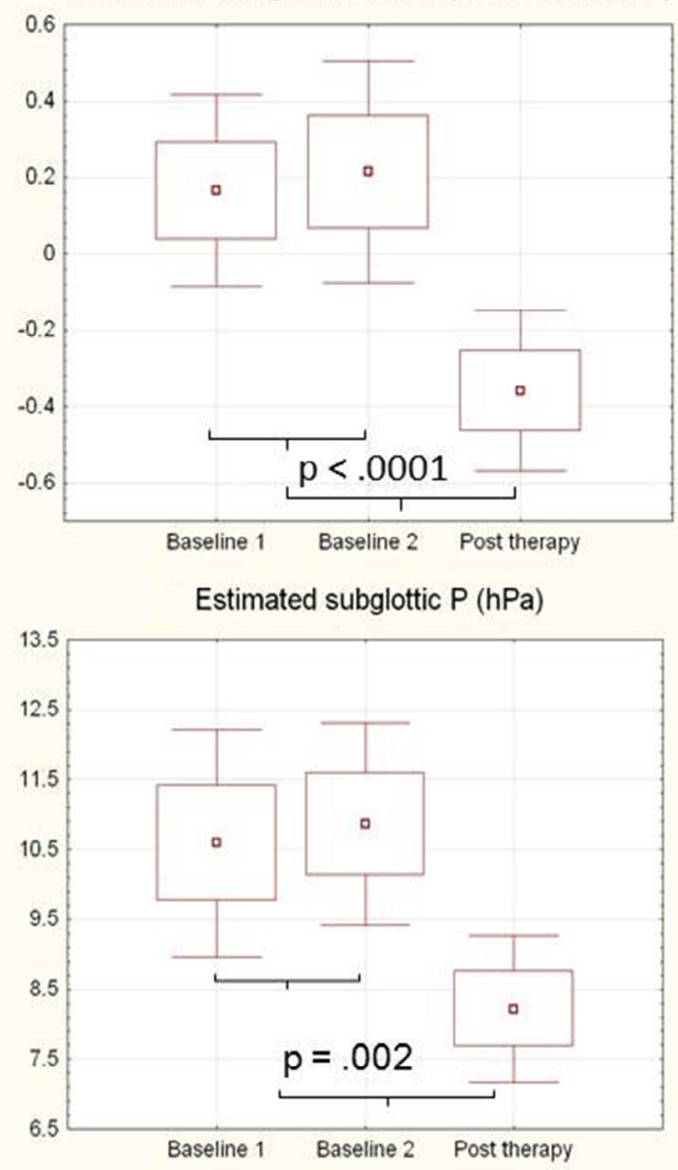

A

I \pm 1.96 std. Err. $\square \pm 1.00^{*}$ Std. Err - Mean

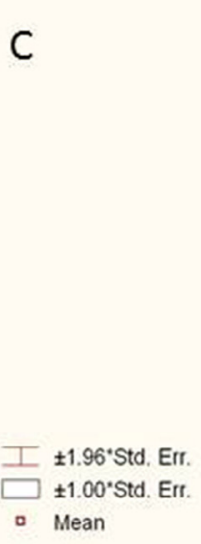

MPT (seconds)

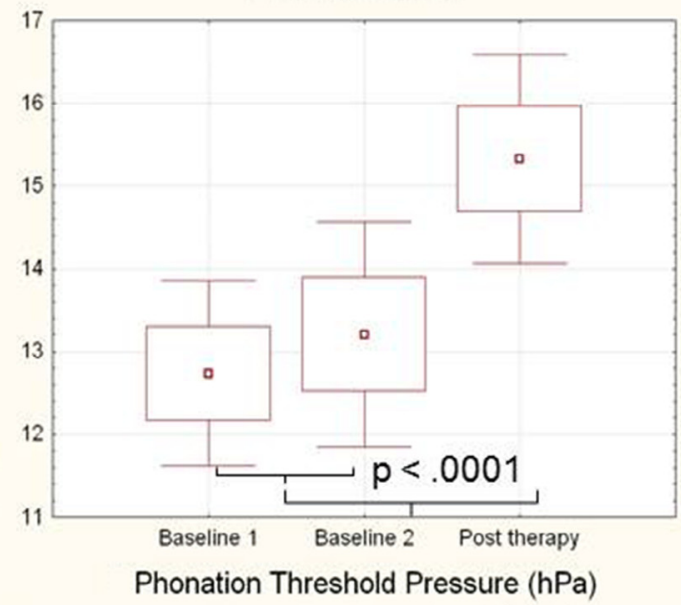

B

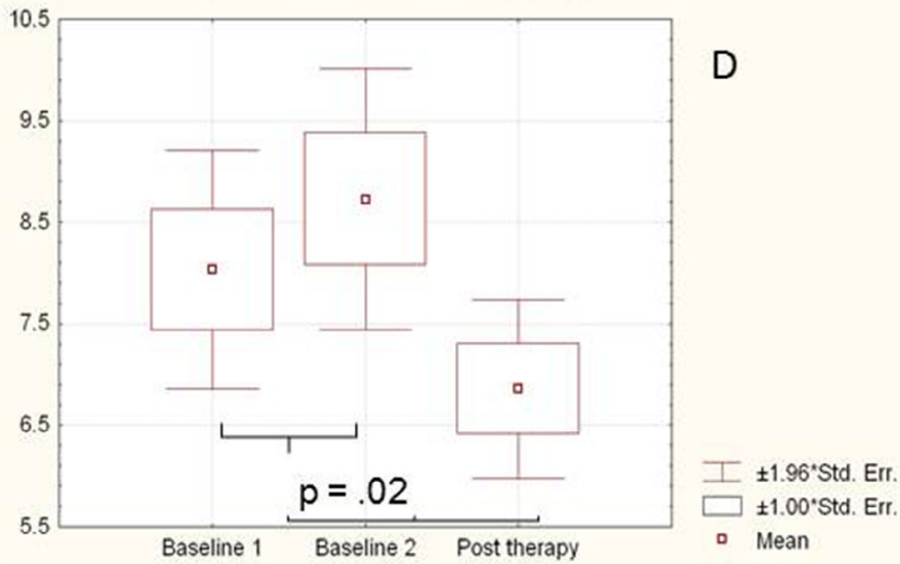

FIGURE 3. Differences between pre- and post-therapy Z-transformed combined aerodynamic scores (A), MPT (B), estimated subglottic pressure $(\mathbf{C})$, and phonation threshold pressure (D). MPT, maximum phonation time; SE, standard error. 


\begin{tabular}{|c|c|c|c|c|}
\hline $\begin{array}{l}\text { Group } \\
\text { No. }\end{array}$ & $\begin{array}{c}\text { No. of } \\
\text { Patients }\end{array}$ & $\begin{array}{l}\text { Mean } \\
\text { Age } \\
\text { (SD), y }\end{array}$ & Diagnosis & $\begin{array}{c}\text { Mean } \\
\text { Elapsed } \\
\text { Time Since } \\
\text { Diagnosis } \\
\text { (SD), y }\end{array}$ \\
\hline 1 & 5 & $42.0(5.5)$ & $\begin{array}{l}2 \text { nodules } \\
2 \text { VFE } \\
1 \mathrm{MT}\end{array}$ & $7.9(6.5)$ \\
\hline 2 & 5 & $39.3(4.9)$ & $\begin{array}{l}1 \text { nodule } \\
2 \text { VFE } \\
2 \text { VFB }\end{array}$ & $10.3(5.2)$ \\
\hline 3 & 5 & $45.6(2.5)$ & $\begin{array}{l}2 \text { nodules } \\
2 \mathrm{VFE} \\
1 \mathrm{MT}\end{array}$ & $8.7(5.4)$ \\
\hline 4 & 5 & $43.6(4.2)$ & $\begin{array}{l}2 \text { nodules } \\
2 \text { VFE } \\
1 \text { VFB }\end{array}$ & $13.6(6.6)$ \\
\hline 5 & 5 & $37.9(5.8)$ & $\begin{array}{l}2 \text { nodules } \\
2 \mathrm{VFE} \\
1 \mathrm{MT}\end{array}$ & $11.6(8.9)$ \\
\hline 6 & 5 & $46.6(4.2)$ & $\begin{array}{l}1 \text { nodule } \\
2 \text { VFE } \\
1 \mathrm{MT} \\
1 \mathrm{VFB}\end{array}$ & $12.1(4.9)$ \\
\hline 7 & 4 & $37.9(6.2)$ & $\begin{array}{l}2 \text { nodules } \\
1 \text { VFE } \\
1 \text { VFB }\end{array}$ & $15.1(6.2)$ \\
\hline
\end{tabular}

$(P<0.0001)$, and that estimated subglottic pressure during phonation and PTP were closely correlated with each other $(r=-0.94)$. Furthermore, the baseline and post-therapy difference in the "above-threshold" phonation pressure is also significant $(P=0.009)$. MPT and estimated subglottic pressure during phonation did not correlate at all (Figure 5).

\section{Correlation between PTP and estimated Psubgl.}

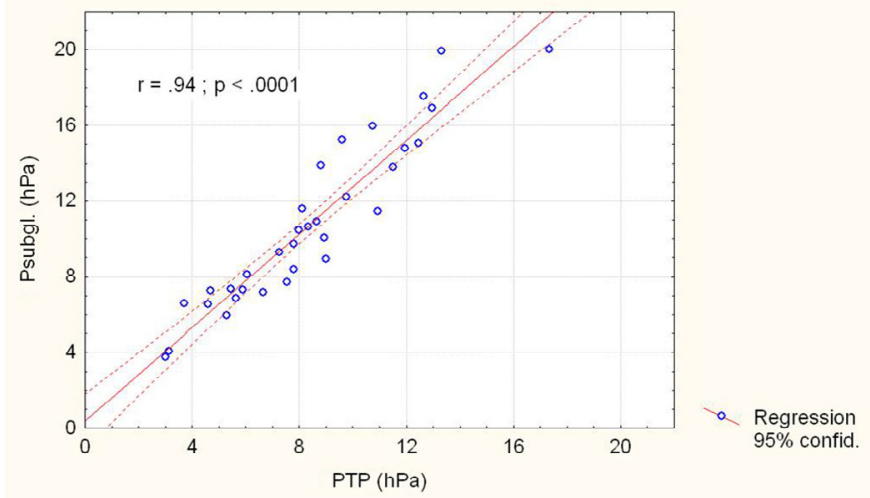

FIGURE 4. Correlation between PTP and estimated Psubgl. Psubgl, subglottic pressure; PTP, phonation threshold pressure.

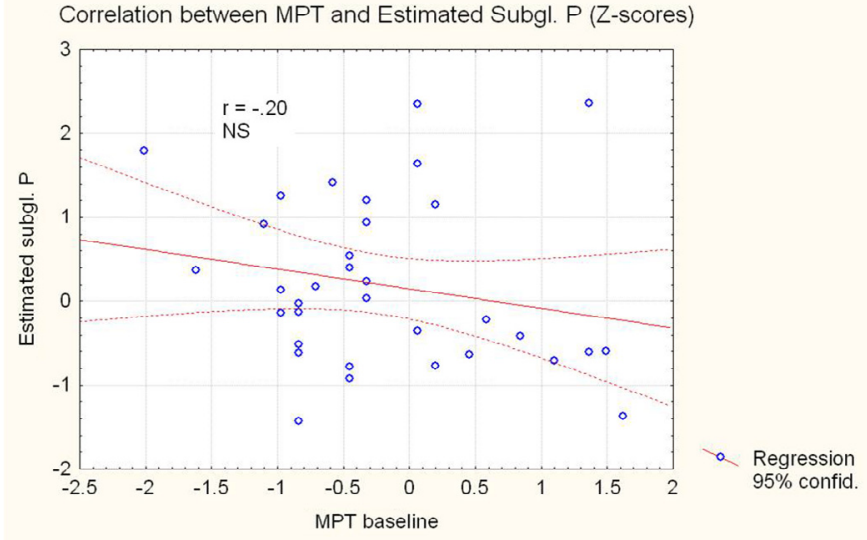

FIGURE 5. Correlation between MPT and Z-transformed estimated Psubgl. MPT, maximum phonation time; NS, not significant; Psubgl, subglottic pressure.

\section{Self-evaluation}

As shown in Figure 6A, the average total VHI score slightly decreased after treatment, but there were large interindividual variations and the comparison of paired values was not statistically significant. However, there was a statistically significant positive difference between the averaged baseline and post-
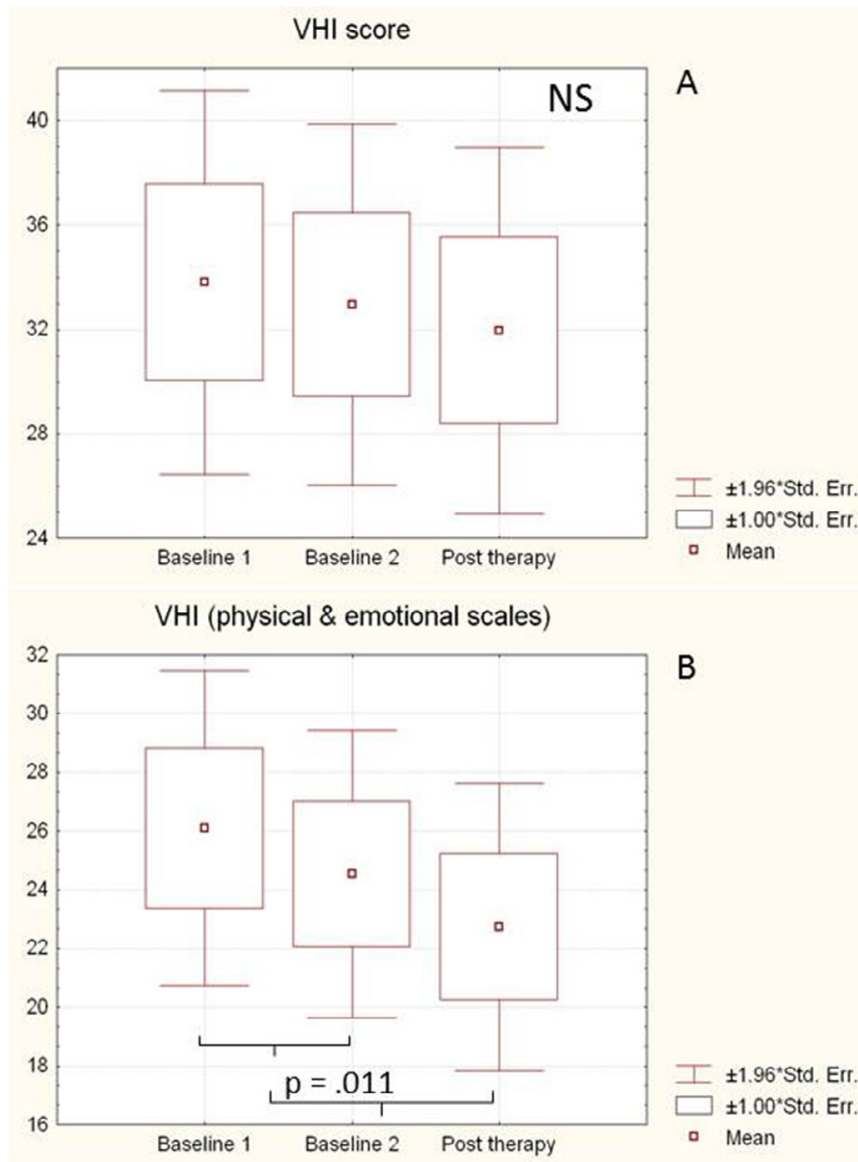

FIGURE 6. Differences between global pre- and post-therapy VHI scores (A) and the VHI physical and emotional subscales (B). SE, standard error; NS, not significant; VHI, Voice Handicap Index. 

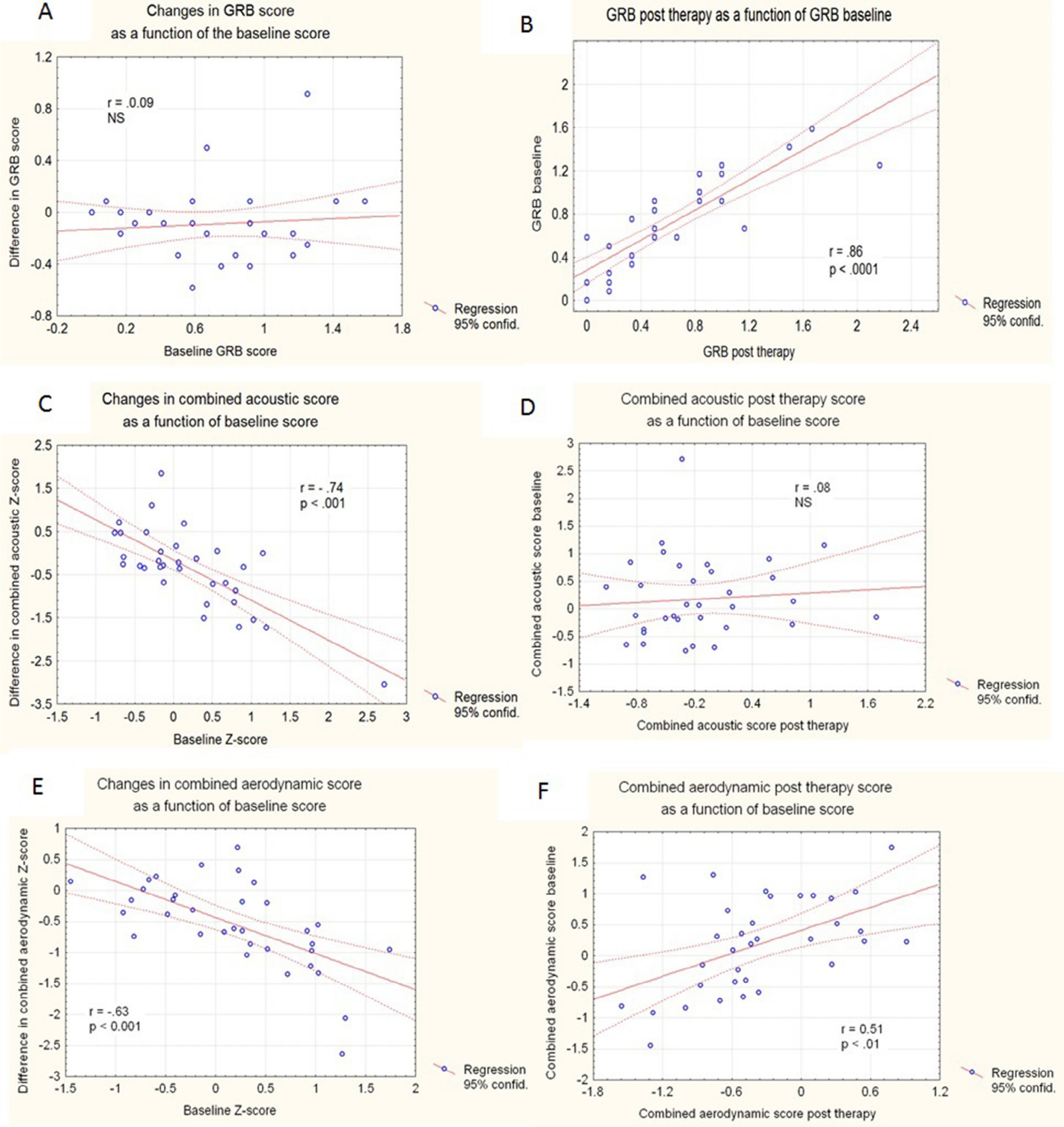

FIGURE 7. A. Changes in GRB scores as a function of baseline scores. B. Post-therapy GRB scores as a function of baseline GRB scores. C. Changes in combined acoustic scores as a function of baseline scores. D. Combined acoustic post-therapy scores as a function of baseline scores. E. Changes in combined aerodynamic scores as a function of baseline scores. F. Combined aerodynamic post-therapy scores as a function of baseline scores. GRB, grade of dysphonia, roughness, breathiness; NS, not significant. 


\section{TABLE 2.}

Cohen's $d$ indices

Dimension/Parameter

Perceptual evaluation (GRB)

Combined acoustic score

Combined aerodynamic score

Maximum phonation time

Estimated subglottic phonation pressure

Estimated phonation threshold pressure

Above-threshold phonation pressure

Self-rated voice-related quality of life (VHI)

Abbreviations: GRB, grade of dysphonia, roughness, breathiness; VHI

Voice Handicap Index.

treatment physical and emotional subscale scores $(P=0.011)$ (Figure 6B).

\section{Effect size}

Table 2 shows the computed values of the Cohen $d$. The aerodynamic dimension obviously showed the strongest effect size but, like that of the acoustic dimension, was "medium." The effect sizes of the perceptual and self-rating dimensions were small.

\section{Correlations between the pre-post differences and dimension redundancy}

Table 3 shows the correlation matrix of the pre-post differences, that is, the differences between the average of the two pretherapy measures (baseline) and the post-therapy measure, in the four dimensions. All of the correlations are weak and none is significant; thus, the dimensions cannot be considered as simply redundant. In other words, an important improvement after therapy in one dimension does not predict an important improvement in the other dimensions, and vice versa.

\section{Correlations between the changes and baseline values}

The magnitude of the post-therapy changes in the perceptual dimension was unrelated to the magnitude of the baseline value (Figure 7A), but the average baseline values were already good (under 1: slight disturbance) (Figure 1). There was a close correlation (Figure 7B) between the baseline and post-therapy values ( $r=0.86, P<0.0001$ ), with a slight shift toward smaller (better) values after therapy. In contrast, the magnitude of the changes in the acoustic parameters was clearly smaller when the degree of baseline abnormality was more pronounced $(r=-0.74, P<0.001)$
(Figures 7C), but the pre- and post-therapy values did not correlate with each other (Figures 7D).

Similarly, the changes in the aerodynamic dimension were generally smaller when the degree of abnormality was larger $(r=-0.63$, $P<0.001$ ) (Figure 7E), but there was a correlation between the baseline and post-therapy values $(r=0.51, P<0.01)$ (Figure $7 \mathrm{~F})$.

The changes in the two most sensitive parameters (MPT and estimated subglottic pressure during phonation) showed a complete lack of correlation and are therefore by no means redundant.

Unlike the objective measures, the self-rated VHI values were comparable with those of the perceptual dimension. For the VHI scores, the magnitude of the post-treatment change was unrelated to the baseline value, but there was a very close correlation between the pre- and post-treatment scores $(r=0.93, P<0.0001)$, with a minimal shift toward a lower score.

\section{DISCUSSION}

The findings of the present study provide new and objective evidence of the effectiveness of group voice therapy in changing vocal fold dynamics in patients with dysphonia due to functional or minor organic changes following phonotrauma. The study subjects achieved a more stable and efficient voice production, with a reduction of phonatory effort post as compared to pretherapy. Only a few studies ${ }^{1,25,26}$ have objectively evaluated the possibility of rehabilitating patients with voice disorders in small groups, and some of these only considered dysphonia in patients affected by Parkinson's disease. ${ }^{25,26}$ Furthermore, to the best of our knowledge, this is the largest study analyzing the effectiveness of group voice therapy that has so far been undertaken.

All of the considered voice dimensions improved, although the improvement in the perceptual evaluation was significant only if the $\mathrm{G}, \mathrm{R}$, and B were grouped and averaged. This finding is presumably because the patients' baseline values were not severely altered as the patients were affected by minor organic changes of the vocal folds or functional dysphonia.

The most important objective of voice therapy in patients affected by dysphonia due to chronic vocal overuse is to reduce often debilitating perceived effort during phonation. The reduction in subglottic pressure during voice production demonstrates that less muscular effort was required to produce vocal sounds. This finding may account, at least to some extent, for a reduction in the patient's perception of phonatory effort. ${ }^{27}$ The improvement in MPT suggests that glottic closure was more efficient as there was less air leakage through the vocal folds during vibration and that an improvement in respiratory control was achieved.

TABLE 3.

Correlation matrix

\begin{tabular}{lcccc}
\hline & $\Delta$ GRB & $\Delta$ Acoustics & $\Delta$ Aerodynamics & $\Delta$ VHI \\
\hline$\Delta$ GRB & 1 & 0.27 & -0.18 & -0.11 \\
$\Delta$ Acoustics & 0.27 & 1 & -0.04 & -0.05 \\
$\Delta$ Aerodynamics & -0.18 & -0.04 & 1 & 0.09 \\
$\Delta$ VHI & -0.11 & -0.05 & 0.09 & 1 \\
\hline
\end{tabular}

Abbreviations: $\Delta$, pre-post difference; GRB, grade of dysphonia, roughness, breathiness; VHI, Voice Handicap Index. 
Furthermore, the significant reduction in the physical and emotional subscales of the VHI shows that voice production was less fatiguing and the emotional aspects of voice had improved post therapy.

Our results are also encouraging in terms of the possibility of maintaining the acquired vocal skills over time insofar as the post-treatment assessment was made 7-10 days after the seventh rehabilitation session, and about 40 days after the completion of the first six sessions.

Our positive findings should stimulate further research into the clinical application of group voice therapy. In very busy practices, the extensive use of group therapy can reduce treatment costs and significantly shorten waiting lists. It can be argued that individual treatment sessions have been proven to be effective and allow more tailored treatment, but we believe that these considerations are counterbalanced by the fact that mutual support and emulation during the sessions contribute to create positive psychological interactions. It is interesting to note that there were no dropouts among the 34 patients, and this may have been favored by group cohesion and motivation. The current literature about the use of group therapy in the field of rehabilitation for neurologic and psychiatric disorders supports our finding that social interaction and competition may create a supportive environment and a favorable condition for achieving positive rehabilitative outcomes. ${ }^{4-6}$

Finally, it has been reported that the lifetime prevalence of voice disorders among teachers is as high as $51.4 \%,{ }^{28}$ and so it is perhaps not surprising that teachers made up the largest occupational group in our study population (38\%). Teachers therefore represent an occupational category that would benefit from the rapid access to treatment that group voice therapy allows.

The main limitation of our findings is that in our open study there was no control group, as each patient was his or her own control. Controlled, randomized studies are needed to confirm our positive findings in the future.

\section{CONCLUSIONS}

Our findings demonstrate the effectiveness of group voice therapy on the basis of the perceptual, acoustic, aerodynamic, and selfevaluation parameters assessed using our multidimensional protocol in a large case series of patients with functional dysphonia and minor anatomic vocal fold pathologies due to chronic vocal overuse. These results prompt us to suggest the usefulness of developing controlled comparative trials of individual and group voice therapies.

\section{REFERENCES}

1. Speyer R, Wieneke GH, Dejonckere PH. Documentation of progress in voice therapy: perceptual, acoustic, and laryngostroboscopic findings pretherapy and post-therapy. $J$ Voice. 2003;18:325-340.

2. Law T, Lee KY, Ho FN, et al. The effectiveness of group voice therapy: a group climate perspective. $J$ Voice. 2012;26:e41-e48.

3. Gomes BC, Abreu LN, Brietzke E, et al. A randomized controlled trial of cognitive behavioral group therapy for bipolar disorder. Psychother Psychosom. 2011;80:144-150.

4. Mannerkorpi K, Gard G. Physiotherapy group treatment for patients with fibromyalgia — an embodied learning process. Disabil Rehabil. 2003;25:1372_1380 .
5. Gauthier L, Dalziel S, Gauthier S. The benefits of group occupational therapy for the patients with Parkinson's disease. Am J Occupational Ther. 1987;41:360-365.

6. De Weerdt W, Nuyens G, Feys H, et al. Group physiotherapy improves time use by patients with stroke in rehabilitation. Aust J Physiother. 2001;47:5361.

7. Hirano M. Psycho-acoustic evaluation of voice: GRBAS scale for evaluating the hoarse voice. In: Hirano M, ed. Clinical Examination of Voice. Vienna: Springer; 1981:81-84.

8. Giovanni A, Robert D, Estublier N, et al. Objective evaluation of dysphonia: preliminary results of a device allowing simultaneous acoustic and aerodynamic measurements. Folia Phoniatr Logop. 1996;48:175-185.

9. Giovanni A, Estublier N, Robert D, et al. Evaluation vocale objective des dysphonies par la mesure simultan é e de param è tres acoustiques et a é rodynamiques à l'aide de l'appareillage EVA. Ann Otolaryngol Chir Cervicofac. 1995;112:85-90.

10. Cantarella G, Baracca G, Pignataro L, et al. Assessment of dysphonia due to benign vocal fold lesions by acoustic and aerodynamic indices: a multivariate analysis. Logoped Phoniatr Vocol. 2011;36:21-27.

11. Smitheran JR, Hixon TJ. A clinical method for estimating laryngeal airway resistance during vowel production. J Speech Hear Disord. 1981;46:138-146.

12. Titze IR. Phonation threshold pressure: a missing link in glottal aerodynamics. J Acoust Soc Am. 1992;91:2926-2935.

13. Neiman GS, Edeson B. Procedural aspects of eliciting maximum phonation time. Folia Phoniatr (Basel). 1981;33:285-293.

14. Jacobson BH, Johnson A, Grywalsky A, et al. The voice handicap index: development and validation. Am J Speech Lang Pathol. 1997;6:66-70.

15. Dejonckere PH. Voice evaluation and respiratory function assessment. In: Anniko M, Bernal-Sprekelsen M, Bonkowsky, V, et al., eds. Otorhinolaryngology, Head \& Neck Surgery. European Manual of Medicine. Berlin: Springer-Verlag; 2010:563-574.

16. Verdolini MK, Bruke MK, Lassac A, et al. Preliminary study of two methods of treatment for laryngeal nodules. $J$ Voice. 1995;9:74-85.

17. Kitzing $P$, Akerlund L. Long-time average spectrograms of dysphonic voices before and after therapy. Folia Phoniatr (Basel). 1993;45:53-61.

18. Murry T, Woodson GE. A comparison of three methods for the management of vocal fold nodules. J Voice. 1992;6:271-276.

19. Dejonckere PH, Bradley $\mathrm{P}$, Clemente $\mathrm{P}$, et al. Committee on Phoniatrics of the European Laryngological Society (ELS). A basic protocol for functional assessment of voice pathology, especially for investigating the efficacy of (phonosurgical) treatments and evaluating new assessment techniques. Guideline elaborated by the Committee on Phoniatrics of the European Laryngological Society (ELS). Eur Arch Otolaryngol. 2001;258:77-82.

20. Cohen J. Statistical Power Analysis for the Behavioral Sciences. 2nd ed. Mahwah, NJ: Lawrence Erlbaum Associates; 1988.

21. Cicchetti DV. Guidelines, criteria, and rules of thumb for evaluating normed and standardized assessment instruments in psychology. Psychol Assess. 1994;6:284-290.

22. Speyer R, Bogaardt HC, Passos VL, et al. Maximum phonation time: variability and reliability. J Voice. 2010;24:281-284.

23. Plexico LW, Sandage MJ, Faver KI. Assessment of phonation threshold pressure: a critical review and clinical implications. Am J Speech-Lan Pathol. 2011;20:348-366.

24. DeJonckere PH. Aerodynamic and acoustic voice measurements (Chapter 12). In: Benninger MS, Murry T, Johns MM III, eds. The Performer's Voice. 2nd ed. San Diego, CA: Plural Publishing Inc.; 2015.

25. Koufman JA, Blalock PD. Functional voice disorders. Otolaryngol Clin North Am. 1991;24:1059-1073.

26. Raabe J, Pascher W. Das Reinke-Odem: eine Untersuchung zu Fragen der Atiologie, der Prognose und der Wirksamkeit therapeutischer Interventionen. Laryngorhinootologie. 1999;78:97-102.

27. Verdolini-Marston K, Sandage M, Titze IR. Effect of hydration treatments on laryngeal nodules and polyps and related voice measures. $J$ Voice. 1994;8:30-47.

28. Angelillo M, Di Maio G, Costa G, et al. Prevalence of occupational voice disorders in teachers. J Prev Med Hyg. 2009;50:26-32. 